\title{
Resolving Security Issues for Semantic Web Related Networks and Datasets
}

\author{
S.Munisankaraiah, $\mathrm{PhD}$ \\ Prof. \& HoD of CSE, Dept of CSE, Dhruva Institute \\ of Engg \& Tech, Near Ramoji Film City, \\ Hyderabad, Telangana State
}

\author{
Kudali Hymavathi \\ System Analyst and Research Scholar, \\ SuchInfotech pvt.Itd, Srinagar colony, Hyderabad, \\ Telangana State
}

\begin{abstract}
Improve the quality of web service composition using the petri nets and ontology. Previously web services are design independently. These kinds of services are not compatible. We observe the different security issues. Now in this paper we design new semantic web networks using petri nets and ontology. Start the interaction process levels display the decisions using colored petri nets environment. After interaction process defines the semantic context information. Control the mismatches mapping decisions information like security issues. It's allows the semantic mapping users as an authentication users. Compare to previous approaches control the more security issues efficiently.
\end{abstract}

Keywords: Semantic web networks, petri nets, ontology mapping procedures, colored petri nets, fuzzy concept.

\section{INTRODUCTION}

Web services those are available freely from over the internet environment. Web services are used in between of different applications. Previous web services applications are independent. Independent web services applications are not efficient. There is no mediator's implementation.

In This paper we design the new semantic web framework using colored petri nets and ontology. Here we use the mediators for exchanges of messages from one place to another place in our framework. Its addresses the semantic context and control the mismatches information. Its solve the security issues efficiently compare to previous approaches.

\section{RELATED WORK}

We address the issues of service description with information related to security constraints and capabilities. We observe the security issues in between users and service providers with present security constraints. Previously different approaches consider the different security constraints for semantic level purpose. Client can take the advantage of security requirements in the communication environment of service provider. Service provider starts the communication with client using SSL support. It does not provide the efficient security. It's possible to provide the efficient security for potential clients.

Some other extra constraints and policies were implemented with the help of semantic web languages. Semantic web languages design the expressive authorization techniques. Again we get the conflict problem in the selection of suitable policy. Resolve the conflict policy selection problem possibly resolve with dynamic policy automatically or suggested policies. We get the good and efficient solutions with adaptive policies in semantic web environment or infrastructure.

There are still some more issues in interaction environment of semantic web architecture.

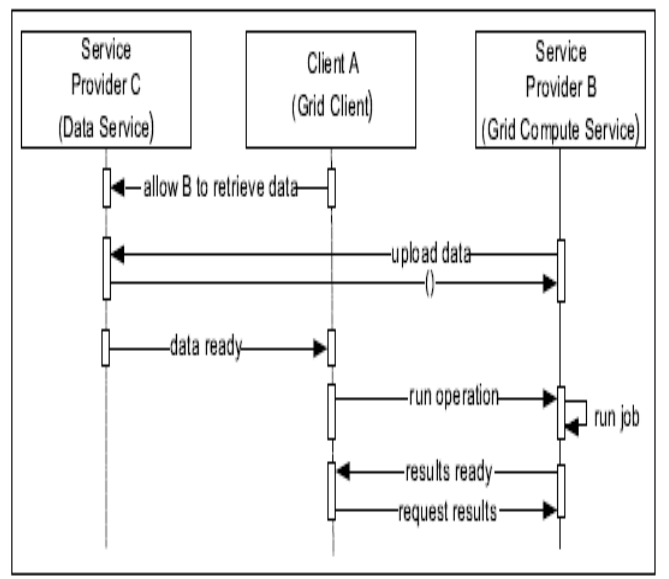

Fig1: Basic Semantic Web Infrastructure Interaction Scenario

Next we provide the security in semantic web using XML security and RDF security concepts. Now we start the investigation for detection of security issues. XML and RDF design the new security standards architecture. Architecture contains different layers. Those layers are logical, proof and trust layers. Using these three layers improve the security. We get the trusted services in different locations like access the documents locations and query/result generation. In some situations issues are generated here.

Next for the semantic web architecture we design the new security infrastructure with different languages. Those languages are XML key management services, security assertion markup language, extensible access control markup language, XML Signature, XML Encryption, Ws- security, federation. Using all those languages enhance the security in semantic web environment.

\section{PROBLEM STATEMENT}

Previously single web service composition is available for resolve the security issues. In order resolve the security issues and improve the quality of web service composition we widely deploy the petri nets and ontology. Petri nets and ontology represents the domain knowledge. These petri nets and ontology we use in the interaction mode in between client and service provider. Taking into account the mediators semantic context services then we resolve the security issues. 
This is completely streamlining process based decision making solution in semantic web networks.

Interaction starts in between client and service provider. Start the exchanges of messages from user to service provider and follow the route. We check the route of intermediate messages. All messages are redirect to a proper order or not we observe in semantic web related networks.

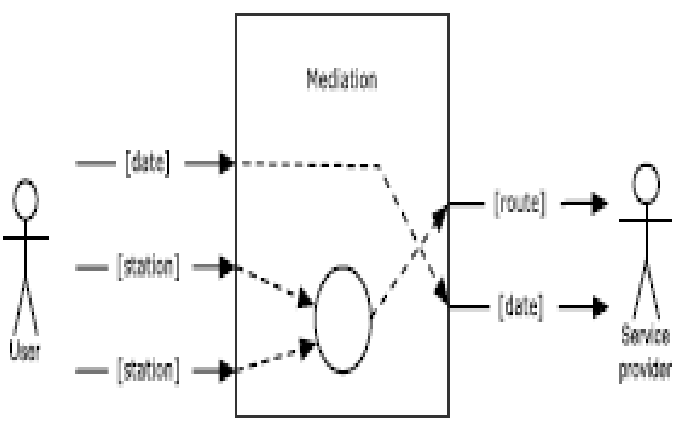

Fig 3: Exchanges messages order

\section{COLORED PETRI NETS}

Ensure the correctness of web service composition we use the WS-BPEL. This WS-BPEL is performing the modeling, analysis and verification.

Consider the inputs like places, transitions, arcs and colors information. Next map operation we perform in between of two places verification transitions information. Finally we produce the expression like true or false.

\section{PROPOSED SYSTEM METHODOLOGY}

Proposed framework designs the semantic and functionality mediator. Define the mediator patterns using BPEL, ontology language for services and petri nets formalisms.

1. BPEL for giving the inputs content

2. $\mathrm{XML}$ for process

3. Petri nets for transformation of content

After completion of the above three steps we check the behavior of patterns. Patterns contain different number of transition states. Analyze the transition states we find out the semantic patterns.

Proposed framework is dividing into different phases

1. Initiation

2. Pre-mediation

3. Mediation execution

4. Final composition

In framework some other external components are used here. Those components are user, service provider and repository.

Mediation execution module performs two operations. Those operations are semantic data mapping and mediator pattern selection. In these operations we specify the input and output operations content. These all inputs provided by the ontology hierarchically. Sometimes it's allows the semantic mismatches and duplicates in the web service interfaces. Here we check the patterns display the semantic matches and mismatched output result. Mismatched states information represents with colored petri nets. Semantic matched users only allow to gets the services.

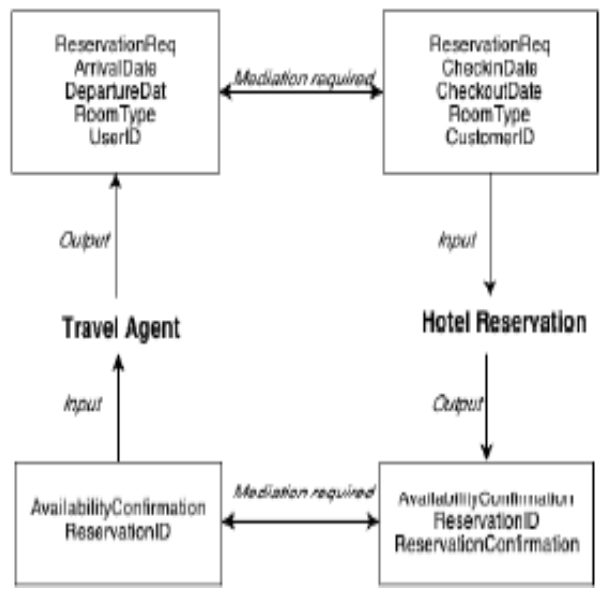

Fig 3: workflow architecture

\section{Merge Mediator Algorithm:}

Input: source, target, mediator, rules

Output: merge the all mediator decisions provide the result as a true or false

Step1: first check the source and target

Step2: agent start the process to reserve the hotel

Step3: mediator apply the rule

Step4: display the status is true

Step5: same way activate the multiple mediators with transform mediator

Step6: end of state merge all rules decisions

Step7: Displays the semantic mapping result are secure

Step8: allows performing the next actions.

\section{VI.EVALUATION AND ANALYSIS}

Here we select the total number of services are six. Using the six numbers of services starts the process. Monitor the workflow using fuzzy concepts environment process. Fuzzy concepts calculate the semantic probability or degree levels. In this process different numbers of components are composed here. Those components are inputs, expected outputs and behavior constraints. The above discussion related different number of service providers and different service requestors. We design the mapping ontology in order to maintain the relationships. Finally derive the results like truth degree and false degree values.

Finally execute the algorithm finally display the probability values. Those probability values are $1,0,0.76,0.68,0.64$, $0.54,1,0,1,0.8,0.8,0.58$.

\section{CONCLUSION AND FUTURE WORK}

In this paper we design the semantic framework using petri nets, ontology and merge meditor algorithm. These three models we used in the interaction, interfacing and processing environment. Using all models generate the semantic mediation and control the security issues. Further we improve the reliability of services distribution using semantic network models procedures or constraints. 


\section{REFERENCES}

[1] T. Berners Lee, J. Hendler, O. Lassila, The semantic web, Scientific American; May 2001, 34 - 43.

[2] What is Social Networking\|, http://www.whatissocialnetworking

[3] Journal of Computer-Mediated Communication http://jcmc.indiana.edu/vol13/issue1/boyd.ellison.html.

[4] Leveraging Social data with Semantics, http://www.w3.org/2008/09/ msnws/papers/ereteo_et_al_2008_leveraging.html.

[5] Virtual economy, WikiPedia, http://en.wikipedia.org/wiki/Virtual_economy.

[6] Burleson, 2007. Introduction to the semantic web Vision and Technologies, http://www.semanticfocus.com/blog/entry/title/introducti on-tothe-semantic-web-vision-andtechnologies-part-2foundations.

[7] B. Matthews, $\|$ semantic web Technologies. JISC Technology and Standards Watch, 2005.

[8] T. Berners Lee, 2006. Uniform Resource Identifiers, URI Generic Syntax. IETF. http://www.ietf.org/rfc/rfc2396.txt. A. Medić, Criptography - Securing web Servers and web Applications, University of Bihać, Technical Faculty Bihać, engineer thesis, Bihać, Bosnia and Herzegovina, February 2008

[9] Fensel, 2002. Layering the semantic web: Problems and Directions. In the Proceeding of 1 st International semantic web Conference (ISWC, 2002). Sardinia, Italy, 9-12 June, pp: 476. ISBN: 3540437606, 9783540437604.
[10] S. St. Laurent, XML, McGraw Hill, New York, NY, 2000.

[11] S. Buraga and G. Ciobanu., 2002. A RDF- based model for expressing spatio-temporal relation between web sites. In The 3rd International Conference on Information Systems Engineering. IEEE Computer Society. pp: 355. IEEE Computer Society Washington, DC, USA. ISBN:0-7695- 1766-8.

[12] Description Frameworkl, in D-Lib Magazine, May.1998; http://www.dlib.org/dlib/may98/miller/05miller.html.

[13] R. Cloran and B. Irwin, 2005. XML Digital Signature and RDF, http://icsa.cs.up.ac.za/issa/2005/Proceedings/ Poster/026_Article.pdf

[14] T. Haytam, Al-Feel, M. Koutb and H. Suoror, semantic web on Scope: A New Architectural Model for the semantic web, Journal of Computer Science 4 (7): 613 $624,2008$.

[15] B. Thuraisingham,W. Ford, Security constraint processing in a distributed database management system, IEEE Transactions on Knowledge and Data Engineering (1995) 274- 293.

[16] Ryan Naraine, -PlayStation Home virtual world hackedll, ZDNET. Dec. 22, 2008. Available: http://blogs.zdnet.com/security/?p=2330.

[17] B. Thuraisingham, Data Mining: Technologies, Techniques, Tools and Trends, CRC Press, Boca Raton, FL, 1998.

[18] B. Thuraisingham, Secure Sematic web Services, Technical Report, University of Texas - Department of Computer Science, 2007. 